\title{
Novel nano-composite multi-layered biomaterial for the treatment of multifocal degenerative cartilage lesions
}

\author{
Elizaveta Kon • M. Delcogliano · G. Filardo • \\ G. Altadonna $\cdot$ M. Marcacci
}

Received: 8 July 2008/Accepted: 27 April 2009/Published online: 26 May 2009

(c) The Author(s) 2009. This article is published with open access at Springerlink.com

\begin{abstract}
We report on a 46-year-old athletic patient, previously treated with anterior cruciate ligament reconstruction, with large degenerative chondral lesions of the medial femoral condyle, trochlea and patella, which was successfully treated with a closing-wedge high tibial osteotomy and the implant of a newly developed biomimetic nanostructured osteochondral bioactive scaffold. After 1 year of follow-up the patient was pain-free, had full knee range of motion, and had returned to his pre-operation level of athletic activity. MRI evaluation at 6 months showed that the implant gave a hyaline-like signal as well as a good restoration of the articular surface, with minimal subchondral bone oedema. Subchondral oedema was almost non-visible at 12 months.
\end{abstract}

Keywords Osteochondral defect · Degenerative lesions · Biomimetic scaffold · Cartilage regeneration

\section{Introduction}

In recent years tissue engineering techniques have emerged as an interesting therapeutic option for the treatment of articular chondral defects with good and potentially durable results. Better results are obtained in the reconstruction of the chondral surface in traumatic lesions, whereas when the osteochondral unit is involved, as in large

E. Kon $(\bowtie) \cdot$ M. Delcogliano · G. Filardo · G. Altadonna ·

M. Marcacci

Department of Orthopaedic and Sports Trauma,

Biomechanics Laboratory, Rizzoli Orthopaedic Institute,

Via Di Barbiano, 1/10, 40136 Bologna, Italy

e-mail: e.kon@biomec.ior.it osteochondral lesions or in case of early degenerative arthritis, the treatment remains a more challenging problem.

Promising results have been obtained with the tissue engineering approach for the treatment of articular cartilage lesions, and nowadays matrix-assisted autologous chondrocyte transplantation is widely used in Europe $[1,6]$.

However, the results obtained are still controversial and osteo-cartilaginous defects or degenerative lesions represent an even more relevant problem in orthopaedic practice $[5]$.

\section{Case report}

The patient was a 46-year-old man complaining of anteromedial left knee pain after playing tennis for the last 6 months. The pain had progressed to the point where he could no longer engage in athletic activity. He reported having undergone ACL reconstruction in the same knee 10 years earlier.

On physical examination there was a mild effusion. The patient's active range of motion was $0-110^{\circ}$, and he was tender on palpation along the medial joint line. Lachman's test was negative. There was no varus-valgus instability.

Plain radiographs showed a $10^{\circ}$ varus deformity and moderate medial joint space narrowing.

Magnetic resonance imaging (MRI) revealed deep and extended osteochondral lesions, involving the medial femoral condyle, the trochlea and the patella.

The chondral lesions were inspected arthroscopically and were grade 4 according to the ICRS classification. The clinical experimentation was approved by the local Ethics Committee. 
Fig. 1 Surgical view of the lesions covered by four patches of the scaffold

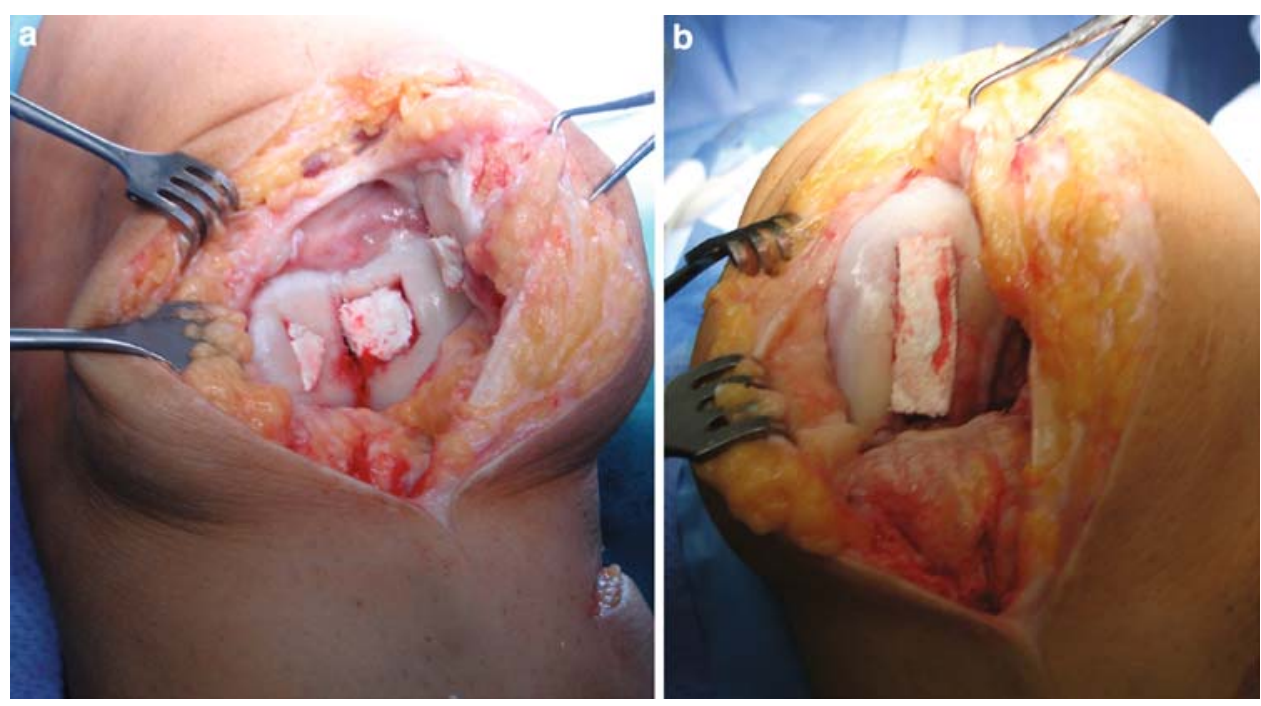

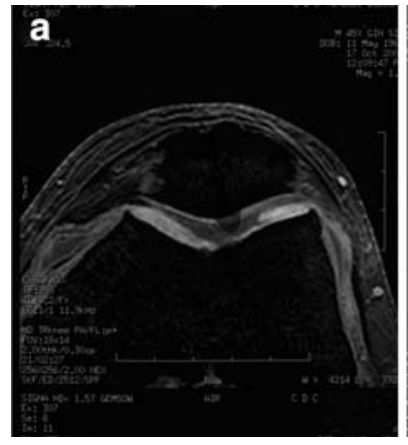
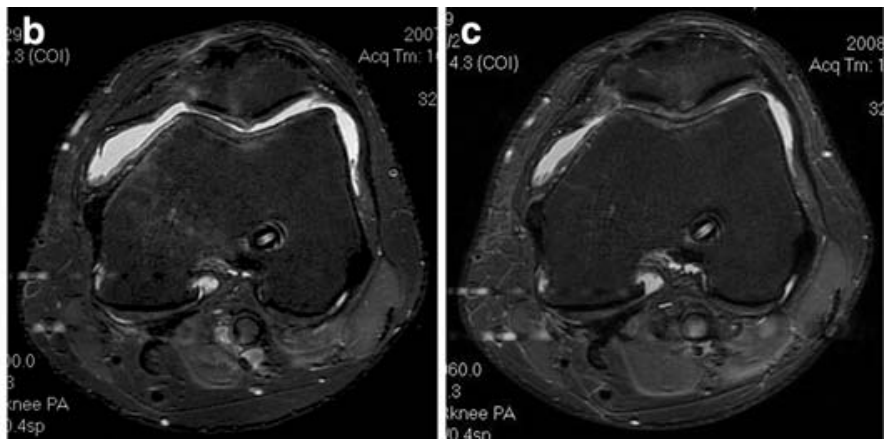

Fig. 2 Axial MRI sequences FSE proton density weighted. a The preoperative MRI shows the trochlea and the patella cartilage defects. b The 6-month follow-up. The MRI shows a continuous and smooth articular surface; two different layers, cartilage and subchondral bone,

A closing-wedge high tibial osteotomy was performed to restore the lower limb normal axis and unload the medial compartment. Next, the biomimetic osteochondral scaffold was implanted to regenerate the damaged articular surface in the following manner.

A medial para-patellar approach was used to expose the lesions. Then, the defects were prepared using a proprietary depth-limiting reamer: the sclerotic subchondral bone was removed to a depth of $9 \mathrm{~mm}$.

The lesions were then sized using templates cut from aluminium foil. The foil templates were then used to prepare the grafts, which were then implanted by a press-fit technique (Fig. 1).

After removing the tourniquet, stability of the implants was tested via cyclic bending of the knee with the grafts under direct visualisation. No weight bearing was allowed for 4 weeks, but isometric quadriceps exercises and continuous passive motion were started on the second postoperative day; initially $0-30^{\circ}$ of motion was allowed, which was increased by $5^{\circ}$ a day. No complications are detectable. Some patella subchondral bone oedema is still present. c The 12-month follow-up. The subchondral bone oedema has decreased, but a subtle effusion is present in the joint

occurred and the partial weight bearing initiated on week 5 arrived to full weight-bearing by week 8 .

Swimming and cycling were allowed at 1 month, low active functional training at 4 months, and repetitive joint impact activities at 10 months.

At 1-year follow-up the patient was pain-free, had full range of motion, and returned to his pre-operation level of tennis.

MRI was performed 6 and 12 months after surgery (Fig. 2).

The implants were stable and showed a hyaline-like signal with good restoration of the articular surface after 6 months. Subchondral oedema progressively decreased over time and at 12 months it was barely evident.

\section{Scaffold structure}

The osteochondral nanostructured biomimetic scaffold (FinCeramica S.p.A., Faenza, Italy) has a porous 3-D tri-layer composite structure, mimicking the whole osteochondral 


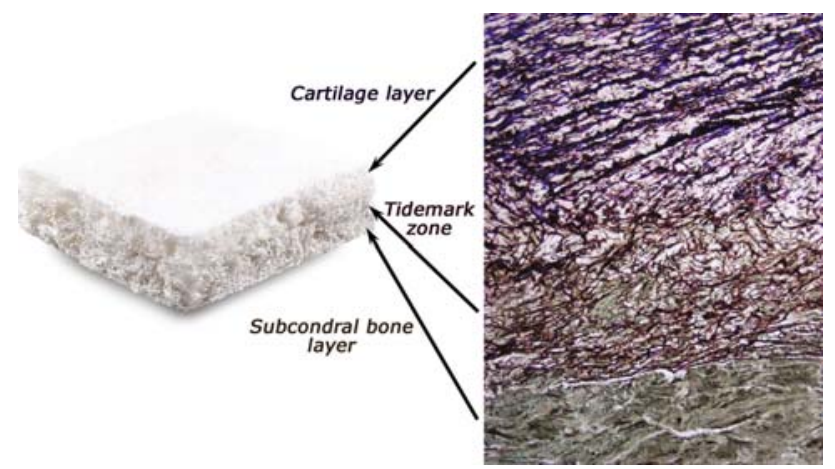

Fig. 3 Macroscopic and histological view of the three-layer nanostructured osteochondral scaffold (Fin-Ceramica S.p.A., Faenza, Italy)

anatomy. The cartilaginous layer, consisting of Type I collagen, has a smooth surface to favour the joint flow. The intermediate layer (tide-mark-like) consists of a combination of Type I collagen (60\%) and HA (40\%), whereas the lower layer consists of a mineralised blend of Type I collagen $(30 \%)$ and HA (70\%) reproducing the sub-chondral bone layer (Fig. 3). Each layer is separately synthesised by a standardised process starting from an atelocollagen aqueous solution $(1 \% \mathrm{w} / \mathrm{w})$ in acetic acid, isolated from equine tendon. The upper non-mineralised chondral layer is of Type I collagen (Opocrin S.p.A., Modena, Italy), the intermediate and the lower layers are obtained by nucleating bone-like nanostructured non-stoichiometric hydroxyapatite into self-assembling collagen fibres, as occurs in the biological neo-ossification process. The final construct is obtained by physically combining the layers on top of a Mylar sheet and finally freeze-dried and gammasterilised at 25 KGray.

\section{Discussion}

The principal finding of the present study was the promising outcome obtained in a patient affected by degenerative cartilage lesion on the medial femoral condyle, trochlea and patella treated with a closed wedge high tibial osteotomy and a newly developed biomimetic nano-structured bioactive scaffold.

For osteochondral defect repair, several authors have highlighted the need for biphasic scaffolds, to reproduce the different biological and functional requirements for guiding tissue regrowth [7]. Following this rationale, in 2007 we started clinical experimentation on a recently
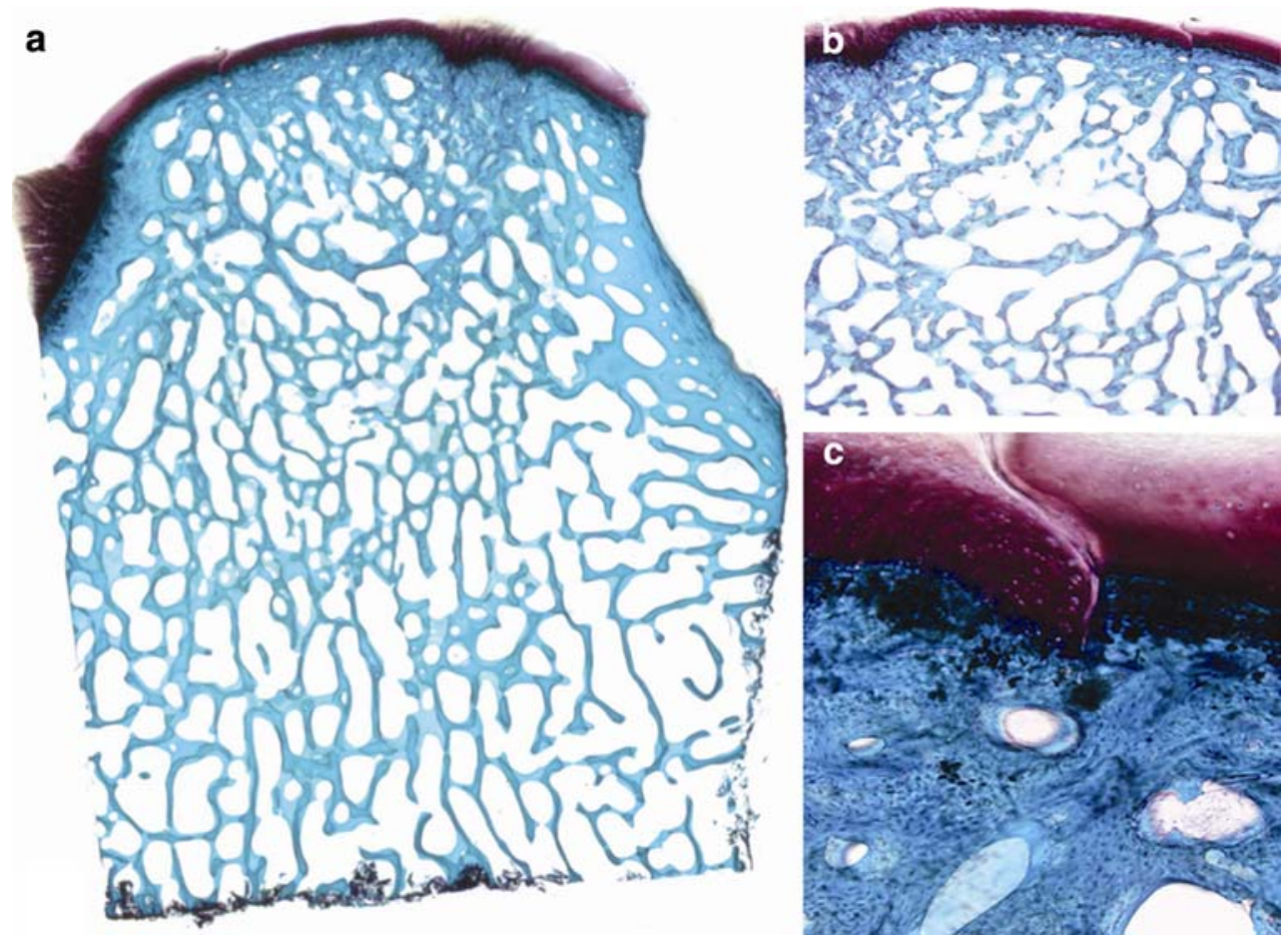

Fig. 4 Histologic evaluation at 6 months of undecalcified samples in a sheep treated with the biomimetic scaffold alone showed the presence of a newly formed repair tissue and a good integration of scaffolds with host cartilage. The presence of newly formed hyalinelike tissue, with a strong proteoglycan staining and columnar rearrangement of chondrocytes, and an underlying well-structured subchondral trabecular bone, distinguishable from the healthy adjacent bone, was noted. Staining with Safranin-O/Fast Green/Toluidine blue $(\mathbf{a} \times 1$ magnification, $\mathbf{b} \times 1.25$ magnification, $\mathbf{c} \times 10$ magnification $)$ 
developed composite scaffold, which mimics the biochemical and biophysical properties of the different layers of native osteochondral structures [8].

In previous in vitro and animal studies (sheep model), we tested this novel biomaterial and obtained good results in cartilage and bone tissue regeneration [3]. In this study of a sheep model we showed the osteochondral regeneration capacity of this 3-layered, biomimetic, nano-structured scaffold, alone or in combination with autologous cells. The 6-month follow-up results indicated poor spontaneous healing of the osteochondral defect in the control sheep group, where the defect, $9 \mathrm{~mm}$ deep and $7 \mathrm{~mm}$ wide, was left untreated. The implant of the gradient biomimetic scaffold led to a reconstruction of both hyaline-like cartilage and structured bone tissue anchored to the interface of adjacent healthy tissues, even with no other bioactive agents added. Healing of the deep osteochondral defect was evident; histological images excluded the presence of bone tissue inside the upper cartilaginous layer and, from a histological viewpoint, no inflammatory reactions were observed in any of the treatment sites (Fig. 4).

Considering that the result was obtained also using the cell-free scaffold, it is likely that mesenchymal precursor cells resident in the subchondral bone mediated this process and recruited within the material. These findings would thus be consistent with previously proposed concepts [2] and a recent study advocating the relevant contribution of mesenchymal stem cells attracted from the bone marrow towards autologous matrix-induced chondrogenesis [4].

This procedure produced good results, both in terms of clinical outcome and MRI findings. The patient was able to return to a satisfactory functional level. We conclude that this scaffold may represent a valid therapeutic option in case of large osteochondral lesions.
Acknowledgments Authors like to thank Angela Montaperrto, Laura Bragonzoni, Biomechanics Lab Rizzoli Orthopaedic Institute; Keith Smith, Task Force Rizzoli Orthopaedic Institute; Clara Montaperto, Marco Nitri: IX Division Rizzoli Orthopaedic Institute; Daniele Pressato, Elena Arcangeli: Fin-Ceramica Faenza S.p.A., Faenza, Italy.

Open Access This article is distributed under the terms of the Creative Commons Attribution Noncommercial License which permits any noncommercial use, distribution, and reproduction in any medium, provided the original author(s) and source are credited.

\section{References}

1. Behrens P, Bitter T, Kurz B et al (2006) Matrix-associated autologous chondrocyte transplantation/implantation (MATC/ MACI) - 5-year follow-up. Knee 13:194-202

2. Buma P, Pieper JS, van Tienen T, van Susante JLC et al (2003) Cross-linked type I and type II collagenous matrices for the repair of full-thickness articular cartilage defects-a study in rabbits. Biomaterials 24:3255-3263

3. Kon E, Delcogliano M, Filardo G, Neri MP, Fini M, Giavaresi G et al (2007) Novel nano-composite biomaterial for ostheocondral tissue engineering. Osteoarthr Cartil 15(Supp 2):B76-B77

4. Kramer J, Böhrnsen F, Lindner U, Behrens P et al (2006) In vivo matrix-guided human mesenchymal stem cells. Cell Mol Life Sci 63:616-626

5. Marcacci M, Kon E, Grigolo B, Filardo G, Delcogliano M, Neri MP (2007) Is osteoarthritis appropriate clinical target for cartilage tissue engineering therapy? The clinician view. Osteoarthr Cartil 15(Supp. 2):B11-B13

6. Marcacci M, Kon E, Zaffagnini S et al (2007) Arthroscopic second generation autologous chondrocyte implantation. Knee Surg Sports Traumatol Arthrosc 15:610-619

7. Martin I, Miot S, Barbero A, Jakob M, Wendt D (2007) Osteochondral tissue engineering. J Biomech 40:750-765

8. Tampieri A, Celotti G, Landi E, Sandri M, Roveri N, Falini G (2003) Biologically inspired synthesis of bone-like composite: self-assembled collagen fibers/hydroxyapatite nanocrystals. J Biomed Mater Res A 67:618-625 